\title{
Les documents cartographiques dans le fonds ancien de l'École des mines de Paris
}

\section{Amélie Dessens}

\section{(2) OpenEdition}

\section{Journals}

Édition électronique

URL : http://journals.openedition.org/artefact/1587

DOI : $10.4000 /$ artefact. 1587

ISSN : 2606-9245

Éditeur :

Association Artefact. Techniques histoire et sciences humaines, Presses universitaires du Midi

Édition imprimée

Date de publication : 30 mai 2018

Pagination : 231-241

ISBN : 978-2-7535-7494-6

ISSN : 2273-0753

\section{Référence électronique}

Amélie Dessens, «Les documents cartographiques dans le fonds ancien de l'École des mines de Paris », Artefact [En ligne], 7| 2017, mis en ligne le 14 février 2019, consulté le 15 septembre 2020. URL : http://journals.openedition.org/artefact/1587

\section{(c) (i) (9)}

Artefact, Techniques, histoire et sciences humaines est mise à disposition selon les termes de la Licence Creative Commons Attribution - Pas d'Utilisation Commerciale - Pas de Modification 4.0 International. 


\section{Les documents cartographiques dans le fonds ancien de l'École des mines de Paris}

Amélie DeSSENS*

Au xviII siècle, alors que l'on cherche à structurer et rationnaliser davantage l'administration des mines en France, la nécessité de repérer et cartographier finement les richesses du sous-sol s'impose. Les premières cartes minéralogiques, notamment l'atlas minéralogique de Guettard et Monnet, sont alors produites. Cette nécessité ne cesse de se renforcer et devient l'une des missions essentielles d'abord de l'Agence puis du Corps des mines qui sera le principal artisan de la carte géologique de France au XIX ${ }^{\mathrm{e}}$ siècle.

Créée en 1794, en même temps que l'Agence des mines et sa Maison d'instruction, la bibliothèque de l'École des mines de Paris conserve dans son fonds ancien un ensemble original de documents cartographiques allant du $\mathrm{XVIII}^{\mathrm{e}}$ à la première moitié $\mathrm{du} \mathrm{xx}^{\mathrm{e}}$ siècle ${ }^{1}$, essentiellement spécialisé en géologie. La présence continue dans un même lieu, sur un peu plus de deux siècles depuis l'installation de l'École à l'hôtel de
Vendôme en 1815, a permis de constituer et conserver un ensemble de documents cohérent et aux formes variées : atlas, cartes monographiques ou en séries bien sûr, mais aussi travaux préparatoires comme les carnets et minutes de terrain, modèles géologiques, exercices d'élèves ou travaux d'ingénieurs en poste.

Ce fonds constitué à l'usage du Corps des mines mais aussi pour servir à l'instruction des élèves reflète l'importance de la carte pour l'ingénieur. Le présent article propose de montrer comment il témoigne alors des activités d'enseignement de l'École tout comme des missions tant professionnelles que scientifiques confiées aux ingénieurs.

\footnotetext{
*. Amélie Dessens est conservatrice des bibliothèques, responsable du pôle Patrimoine de la bibliothèque de l'Ecole des mines de Paris depuis juillet 2016. Elle a été auparavant adjointe à la responsable de la bibliothèque de l'Institut de géographie, département de la bibliothèque interuniversitaire de la Sorbonne (BIS), en charge de la valorisation du fonds cartographique à travers notamment le projet de catalogage rétrospectif. Contact : [amelie.dessens@mines-paristech.fr].
} 


\section{Origine et constitution du fonds}

L'acquisition de documents cartographiques est attestée dès les origines de la bibliothèque. La nécessité de repérer géographiquement les ressources exploitables est en effet directement liée à la mission confiée au Corps des mines alors que la carte est devenue à partir $\mathrm{du} \mathrm{xvIII}^{\mathrm{e}}$ siècle un outil indispensable d'aide à la décision administrative et d'aménagement du territoire ${ }^{2}$. Cette mission exige donc d'avoir à disposition des fonds de cartes topographiques fiables sur lesquels sont reportées les observations de terrain. Ce sont les cartes de Cassini, mais aussi de Chanlaire, qui vont longtemps remplir ce rôle et on sait, grâce aux archives conservées à la bibliothèque notamment, que ces cartes sont régulièrement empruntées par les ingé-

Au-delà de ces indications, il est très difficile d'attester de la continuité des collections cartographiques entre la première bibliothèque et celle que nous connaissons aujourd'hui. Les premiers livres d'inventaire à notre disposition, source quasiment unique parmi les archives de la bibliothèque, ne débutent qu'en 1844. Or, entre 1794 et 1815, les déménagements successifs de la Maison des mines depuis l'hôtel de Mouchy vers l'hôtel du Petit-Luxembourg puis vers l'hôtel de Vendôme, ne permettent pas de s'assurer de la continuité exacte des collections. Quant aux documents achetés entre 1815 et 1844 , comment en dresser une liste certaine en l'absence d'éléments de preuve?

On pourra cependant remarquer que les principaux documents qui, entre la création de l'École et son installation

à l'hôtel de Vendôme, auraient pu être utiles aux missions des ingénieurs et à l'instruction des élèves, sont bien effectivement présents dans la réserve. On trouve ainsi pour les fonds topographiques, la carte de Cassini (1744-1787, $1^{\text {re }}$ édition), la carte réduite de la France par L. Capitaine (1789-1790), la carte physique et hydrographique de la France par Dupain-Triel (1782), ou encore l'Atlas de la partie méridionale de l'Europe, par P.G. Chanlaire $(1801)^{4}$. On trouve également les premiers essais de cartes ou atlas thématiques sur les ressources minéralogiques: l'Atlas minéralogique de la France par A.G. Monnet et J.E. Guettard (1780) (Fig. XXV, cahier couleur) ou encore la Carte géographique et minéralogique de la route de Brest à Paris et de Paris à Tobolsk en Silésie par J. Chappe d'Auteroche (1769).

Parmi les cartes anciennes, les références ne sont pas limitées à l'espace national. On remarque ainsi quelques cartes antérieures à 1800 concernant l'Allemagne (1784), l'Espagne (17651798), l'Italie (carte par le Sieur d'Anville [1743], des plans de Gênes en 1789 ou de Venise en 1787), les Pays-Bas autrichiens en 25 feuilles par le comte de Ferraris (1770-1778) ou encore la Russie (1787) et la Suède (1792). Signalons aussi qu'un travail de dépouillement des cartes comprises dans les documents anciens, dont principalement les récits de voyage, a été mené par la bibliothèque ${ }^{6}$, cartes qui complètent avantageusement la collection. Au total on peut signaler près de 750 cartes et une vingtaine d'atlas édités avant 1844 , date du premier livre d'inventaire. 
Après cette date, les informations sont plus nombreuses pour reconstituer l'histoire du fonds. Les cartes, inscrites à l'inventaire général des documents entrés à la bibliothèque, proviennent à la fois d'achats chez des éditeurs distributeurs à Paris tels qu'Andriveau-Goujon, ou encore Klincksiek, mais elles sont aussi très souvent acquises par don. La bibliothèque reçoit ainsi des livraisons des cartes topographiques d'état-major provenant régulièrement du Dépôt de la guerre, des cartes marines ou hydrographiques du ministère de la Marine, ou encore des cartes géologiques départementales du ministère des Travaux publics. Le ministère des Affaires étrangères adresse parfois également les cartes qu'il reçoit des gouvernements étrangers en rapport avec les disciplines enseignées à l'École, notamment la géologie : en 1853, il est fait par exemple mention de la Geognostische Karte Tirols (1849), envoyée par le gouvernement autrichien ou encore de cartes du Geological survey of Great Britain, par le gouvernement britannique. Le directeur des Ponts et Chaussées, Minard, donne aussi un ensemble de 64 cartes qu'il a réalisées sur les mouvements des combustibles minéraux ou la circulation des marchandises par voie d'eau et de fer. L'École des mines de Paris est donc bien identifiée parmi les cartothèques spécialisées en géologie aux côtés d'autres grandes institutions comme le Museum $d^{\prime}$ Histoire naturelle, et en exploitation des ressources minières. Elle reçoit par ailleurs des dons ou échanges de la part d'autres institutions de recherche étrangères. La Smithsonian Institution, nouvellement créée à Washington en 1846, envoie des cartes des différents États américains. L'établissement géographique de Bruxelles, organisme privé spécialisé dans la production cartographique, envoie des cartes administratives et industrielles ou encore des cartes des concessions houillères de Belgique en échange de numéros des Annales des mines. Les échanges se font également avec l'École de Freiberg, l'une des toutes premières écoles des mines créées en Europe au $\mathrm{xvIII}^{\mathrm{e}}$ siècle. Ces échanges prouvent l'insertion de l'École dans les réseaux scientifiques internationaux, tout comme le montre aussi dès 1839, la circulation des travaux d'Élie de Beaumont et Dufrénoy qui, compilés avec ceux de L. von Buch et G. B. Greenough, a permis la réalisation de la carte géologique de l'Allemagne, la France et l'Angleterre par H. von Dechen?

Enfin, un dernier ensemble de donateurs est formé par les professeurs et anciens élèves de l'École comme Dufrénoy, Élie de Beaumont, Chancourtois, Rivot $^{8}$, etc. Comme pour les livres, cette pratique rappelle l'attachement à l'établissement et sa fonction patrimoniale et identitaire au sein du Corps, déjà très présente. C'est aussi un moyen de diffuser ses travaux auprès de spécialistes dont on sait qu'ils fréquentent la bibliothèque. On trouve ainsi ceux de F. de Botella, ancien élève étranger, sur la géologie de l'Espagne et du Portugal ${ }^{9}$ ou de J. Marcou sur la géologie des États-Unis où il s'est installép ${ }^{10}$.

Une note dans les archives concernant la préparation du catalogue imprimé de la bibliothèque en 1898, fait état de 1253 titres de cartes allant du XVIII ${ }^{\mathrm{e}}$ à la fin du XIX ${ }^{e}$ siècle. L'ensemble a continué de s'enrichir ensuite des cartes les plus récentes publiées sur les sujets de prédi- 
lection de l'École. On note en effet qu'il y a, en moyenne, rarement plus de trois à quatre ans entre la publication du document et son acquisition : la cartothèque est un outil directement lié à l'enseignement, aux recherches en cours et aux travaux des ingénieurs. La bibliothèque compte ainsi l'Atlas souterrain de la ville de Paris (1859), mais aussi bien sûr toute la série des cartes géologiques détaillées de la France qui étaient fournies sans doute directement par le Service de la carte géologique installé également à l'hôtel de Vendôme. Par ailleurs, on note que si une grande majorité des cartes sont topographiques et géologiques, les cartes thématiques sur d'autres sujets sont de plus en plus nombreuses au cours du XIX ${ }^{e}$ siècle et touchent entre autres l'hydrographie, l'hydrogéologie, les reliefs et systèmes de montagne, l'industrie avec les cartes statistiques entre autres, les gites minéraux ou encore les voies de communication dont les chemins de fer, alors que le Corps des mines se voit confier la responsabilité du " matériel roulant» par une ordonnance de $1846^{11}$.
Enfin, du point de vue de la couverture géographique, le fonds n'est pas limité, loin s'en faut, au territoire hexagonal. La constitution de l'empire colonial au XIX ${ }^{e}$ siècle s'est aussi accompagnée d'une exploration des nouveaux territoires conquis et bientôt de recherches sur leurs géologies et ressources exploitables. Les éditions des cartes issues de ces travaux souvent menés par des ingénieurs des mines ont été déposées à la bibliothèque.

Au-delà, l'intérêt pour les pays étrangers est très perceptible. En dehors des cartes géologiques de la France, les cartes sont conservées encore au début du $\mathrm{xx}^{\mathrm{e}}$ siècle dans huit tiroirs par aires géographiques: Italie et Espagne, Amérique (cartes géologiques), Amérique (cartes topographiques et diverses), Europe du Nord, Allemagne - Saxe - Autriche, Angleterre, Ecosse et Irlande, et Afrique - Asie - Australie. Cela révèle à la fois une volonté d'observer comment fonctionnent les administrations similaires dans les autres pays, mais aussi un intérêt scientifique et de recherche englobant toutes les régions du monde.

\section{La cartographie dans l'enseignement de l'École des mines de Paris}

Parmi les fonds cartographiques conservés dans la réserve de la bibliothèque, on distinguera ceux directement liés à l'activité d'enseignement de l'École. Il s'agit avant tout d'un fonds manuscrit issu des exercices proposés aux élèves.

La cartographie est un élément important du métier d'ingénieur comme nous le verrons plus bas. Il est donc logique de la voir figurer parmi les enseignements dispensés à l'École des mines de Paris comme elle l'est dans d'autres écoles d'ingénieurs telles que l'École polytechnique ou celle des Ponts et chaussées ${ }^{12}$, bien qu'elle ne semble cependant pas tout de suite occuper la même importance dans les programmes de cours. Le lever de plan est d'abord enseigné 
au sein du cours d'exploitation des mines, sous la responsabilité notamment de J.P.F. Guillot Duhamel père dès 1783, dans la première école des mines fondée sous l'Ancien régime, ou encore de Ch. Combes, de 1832 à 1844. Ce n'est qu'après cette date que les leçons de topographie sont distinguées de ce cours et prennent leur autonomie ${ }^{13}$. Le programme des cours de 1869 donne le détail de ces leçons qui comprenaient une partie sur le lever superficiel, une autre sur le lever souterrain, et in fine, une partie réservée aux applications de ces leçons : détermination et tracé $d^{\prime}$ 'une méridienne, d'une longitude, ou encore tracé d'un cours d'eau ${ }^{14}$.

Un petit ensemble de plans de quartiers, de cours d'eau ou de carrières, levés lors d'exercices de topographie à Paris ou dans ses environs, est encore conservé à la bibliothèque ${ }^{15}$. Le nombre relativement limité de ces documents - environ une quinzaine datés de 1848 à 1868 - indique qu'ils étaient probablement gardés par les élèves à l'issue $\mathrm{du}$ cours. L'origine du fonds actuel n'est pas connue. Les exercices étaient pratiqués par «brigades » ou groupes d'élèves dont les statuts étaient différents : élèves ingénieurs, élèves titulaires ou encore élèves étrangers, tous participaient à ces exercices sur le terrain. Les titres des exercices renseignent à la fois sur les lieux étudiés mais aussi sur les techniques et instruments de mesure employés alors, plus ou moins sophistiqués : levers à la planchette, à la boussole et au niveau ou encore au théodolite. Ces termes font d'ailleurs écho à la collection d'instruments topographiques conservés à l'École des mines ${ }^{16}$, acquis pour les besoins des laboratoires et des ensei- gnements donnés à l'hôtel de Vendôme. La finesse et l'élégance du dessin, souvent délicatement aquarellé, rappellent son importance dans la formation de l'ingénieur.

Dessin et lever de plan étaient ensuite directement mis à profit dans les journaux ou mémoires de voyage des élèves, très souvent richement illustrés. Cet ensemble original qui couvre presque tout le $\mathrm{xIX}^{\mathrm{e}}$ siècle, de 1824 aux années 1920, compile près de 2000 rapports d'élèves envoyés sur le terrain, seuls ou à plusieurs, pour visiter des sites miniers ou industriels et étudier aussi la géologie d'une région. Les élèves étaient envoyés partout en France mais aussi en Europe et jusqu'en Russie, en Afrique du Nord ou en Amérique. Une carte hydrographique de la France, donnée par le ministère des Travaux publics fut même spécialement réservée pour y faire figurer les parcours des élèves dans les années 1850. Ces documents sont des sources précieuses pour l'industrie et les mines des nombreuses régions visitées, parfois même les seules lorsque les entreprises ellesmêmes ou leurs archives ont disparu.

Ces mémoires présentent un certain nombre de cartes pour servir différents objectifs. Les cartes pouvaient servir par exemple à situer les différents lieux visités, en introduction ou en fin de rapport. Reproduites sur des calques, elles pouvaient servir de fond topographique sur lequel reporter les observations géologiques, ce qui fait aussi écho aux courses organisées par ailleurs par l'École, durant lesquelles les élèves étaient initiés à la géologie de terrain. Henri Peslin propose ainsi une carte géologique du bassin houiller du sud du Pays de Galles dans son mémoire sur la 
production de fonte dans cette région, en $1857^{17}$.

Arrêtons-nous sur le Journal de voyage de MM. J. Roche et A. Badoureau en France, Espagne, Portugal et Algérie (1877) ${ }^{18}$, particulièrement révélateur des différents usages de la carte dans ce type d'exercice. On trouve ainsi page 6 l'itinéraire emprunté par les deux élèves à travers les quatre pays traversés. Une carte imprimée de l'Espagne et du Portugal, éditée par Fremin, géographe à Paris, sert ensuite de fond topographique pour représenter la géologie de la péninsule ibérique, avec des aplats de couleur ajoutés à la main, légendés en haut à gauche. De nombreux calques reproduisent des cartes thématiques comme les chemins de fer en Espagne, avec des détails remarquables comme le tracé de la ligne qui traverse la chaîne cantabrique et l'indication des cotes au-dessus de la mer. Pages 148-149, les élèves dressent le plan général des mines de Bilbao au $1 / 40000^{\circ}$, puis celui des mines de Tafna, pages 360-361. Enfin, mais la liste n'est pas ici exhaustive, à la page 409 (Fig. XXVI, cahier couleur), une carte de France dessinée par Badoureau, représentant en grisé les départements qui n'ont pas produit de fonte entre 1870 et 1872 et en couleur les importations de ce même produit selon son origine, montre un autre degré de réflexion : il ne s'agit plus ici d'une carte pour situer mais d'une carte pour démontrer à partir de données statistiques, l'état économique du pays.

Bien d'autres cartes sont présentes dans ces journaux et mériteraient une étude d'ensemble approfondie. L'École des mines s'attache aujourd'hui à décrire précisément ces documents dans le catalogue Calames ${ }^{19}$ et à mettre à disposition des chercheurs leur numérisation au sein de sa bibliothèque patrimoniale numérique $^{20}$. Une étude sur les cartes qu'ils renferment serait très utile pour mieux cerner quels étaient les savoirs cartographiques enseignés à l'École et complétés par les visites de terrain, ou encore pour observer quelle était la typologie des cartes à disposition des ingénieurs et surtout l'évolution ou la permanence de leurs usages tout au long du XIX siècle. Nous avons en effet ici, même s'il s'agit de travaux d'élèves, un ensemble de cartes important, imprimées ou manuscrites, levées d'après le terrain ou reproduites de documents déjà existants, qui nous sont parvenues directement en lien avec le contexte dans lequel elles ont servi, particularité suffisamment précieuse pour être signalée.

\section{Les cartes entre outils de travail et projet scientifique}

Un arrêté de 1794 concernant les missions des ingénieurs des mines précise déjà que ces derniers sont chargés de repérer les substances minérales de l'arrondissement dans lequel ils sont envoyés, de " tracer sur des cartes les découvertes qu'ils feront ", ainsi que de lever les plans des mines visitées ${ }^{21}$. En 
1810, le décret d'organisation du Corps impérial des mines indique quant à lui, $\mathrm{qu}^{\prime}$ « il sera fait un inventaire détaillé de tous les plans, papiers et cartes, et des instruments appartenant à l'État, existant dans les bureaux des ingénieurs en chef et des ingénieurs ordinaires ». La carte est donc un instrument de travail quotidien pour l'ingénieur des mines dès les origines. Notons qu'il s'agit d'un outil destiné au service de l'administration pour la gestion du territoire mais aussi d'un instrument grâce auquel l'ingénieur peut faire progresser les connaissances scientifiques des terrains dont il a la charge et les diffuser. Cette double mission administrative et scientifique se retrouve dans le fonds cartographique de la bibliothèque de l'École des mines, les deux étant le plus souvent indissociables.

L'un des documents les plus remarquables du fonds se trouve être l'Atlas des concessions houillères des mines de la Sarre (Ms 43) ${ }^{22}$. Ce document manuscrit réalisé en 1810 a été constitué sous la direction de deux ingénieurs des mines, L. Beaunier et M. Calmelet. Envoyés dans les territoires nouvellement conquis par $l^{\prime}$ Empire, ils ont accompli en très peu de temps un travail de repérage des gisements et de découpage de cet espace en 60 concessions minières qui devait servir à leur attribution, selon la législation tout juste établie pour la gestion des ressources minières par l'État ${ }^{23}$. Présenté à l'empereur Napoléon en 1812, l'atlas richement relié et finement aquarellé (Fig. XXVII et XXVIII, cahier couleur) est un exemple du type de travail cartographique qui pouvait être confié à la réalisation des ingénieurs pour l'inventaire des richesses d'une région et leur administration. Hautement stratégique pour un tel espace frontalier, il fut caché par Théodore de Gargan, ancien élève et collaborateur de Beaunier en 1814 face à l'avancée des Prussiens. Réclamé au moment du Congrès de Vienne par ces derniers à qui vient d'être attribuée la Sarre, il ne sera rendu à la France qu'après le traité de Versailles en 1919 et déposé par M. Sainte-Claire Deville au nom de M. Defline, directeur des mines de la Sarre, en 1924 à la bibliothèque de l'École des mines, "sanctuaire digne de le conserver ${ }^{24} »$. Ces mots sont très éloquents quant à la valeur du fonds pour le Corps des mines. La fermeté avec laquelle le directeur de l'École, Gabriel Chesneau, s'oppose l'année suivante à son transfert aux Archives nationales à la demande du ministère de l'Instruction publique et des beaux-arts ${ }^{25}$, prouve combien au-delà de son utilité pratique et administrative au moment de sa réalisation, l'atlas a acquis une valeur historique et patrimoniale très forte. Son dépôt à l'hôtel de Vendôme renforce par effet de miroir le caractère patrimonial et identitaire du fonds lui-même.

Par ailleurs, au cours du xix siècle, l'administration des mines a participé très activement à trois entreprises cartographiques d'ampleur nationale, quand elle n'en a pas été l'initiatrice : la carte géologique de France, les cartes géologiques départementales et la carte géologique détaillée de la France. Le fonds ancien de la bibliothèque permet de bien appréhender le contexte matériel, administratif et scientifique, et les enjeux de la création de ces cartes.

Dans la première moitié du xIx $x^{e}$ siècle, la France accuse un certain retard dans l'étude des sous-sols : l'Angleterre 
surtout, a déjà à disposition une carte géologique avec la "Grande carte » au $1 / 300000^{e}$ réalisée par William Smith ${ }^{26}$ ou encore celle de G.B. Greenough de 1820, dont la bibliothèque possède un exemplaire. Seuls les environs de Paris ont bénéficié d'une description plus précise, avec les travaux d'A. Brongniart et G. Cuvier ${ }^{27}$. En 1822, on confie alors à A. Brochant de Villiers, professeur de l'École, la réalisation d'une carte géologique de la France, carte qu'il réalisera avec le concours de deux ingénieurs, A. Dufrénoy et L. Élie de Beaumont (Fig. XXIX, cahier couleur). Les archives de ce dernier (Ms 65), achetées par $l^{\prime}$ École en $1907^{28}$, permettent de suivre la démarche de l'entreprise : d'abord envoyés en Angleterre « recueillir divers documents sur les mines et particulièrement les notions qui peuvent être utiles à l'exécution ultérieure de la carte géologique de France ${ }^{29}$ ", chacun des deux ingénieurs est en charge d'une partie du territoire national. Élie de Beaumont est plus particulièrement chargé des départements de l'Est et de l'étude du "Système des Alpes ${ }^{30}$ ». Son supérieur l'invite à le tenir au courant de l'évolution de ses recherches tous les quinze jours et à collecter des échantillons, dont des fossiles, pour la collection de l'École : un travail colossal dont nous gardons aujourd'hui la trace grâce aux carnets de terrains et aux catalogues des envois à l'École correspondant aux collections du musée. La carte géologique de France ne fut présentée à l'Académie des sciences qu'en 1835, puis 1840 avec la figuration du relief $\mathrm{f}^{31}$, tandis que $l^{\prime}$ Explication de la carte géologique de la France ne fut publiée qu'à partir de 1841. Alors que la géologie est en train de se structurer en tant que science, l'entreprise de la Carte géologique permet aux savants français, parmi lesquels nombre d'ingénieurs du Corps des mines, de se positionner parmi ses plus éminents spécialistes, à la suite des précurseurs, tels Dolomieu, professeur à l'École des mines au tournant des $\mathrm{XVIII}^{\mathrm{e}}$ et $\mathrm{XIX}^{\mathrm{e}}$ siècle. De 1794 à 1914, 23 des 58 membres de l'Académie des sciences dans la section géologie et minéralogie appartiennent au Corps des mines, dont une grande majorité sont professeurs à l'École ${ }^{32}$.

"Monument pour la science ${ }^{33}$ ", complétée par les informations fournies par les ingénieurs stationnaires sur les mines et usines ${ }^{34}$, la carte au $1 / 500000^{e}$ n'est cependant pas suffisante pour une connaissance précise des ressources minières et des terrains de chaque localité, laquelle doit servir avant tout les intérêts économiques du pays et le développement tant de l'industrie minière que des exploitations agricoles ainsi que celui des chemins de fer et des voies de communication. Dès 1835, A. Legrand, sous-secrétaire d'État chargé des travaux publics, invite les préfets à faire réaliser, avec le concours des ingénieurs des mines en poste dans les départements et des personnes volontaires, des cartes géologiques départementales. Seuls quelques départements avaient déjà fait réaliser ce type de cartes comme la Corse (1820) ou encore le Calvados (1829). La bibliothèque de l'École des mines conserve actuellement près de 130 de ces cartes, accompagnées très souvent de coupes des terrains étudiées, mais aussi de mémoires pour l'explication des cartes et de statistiques départementales $^{35}$. Ces cartes ne sont pas uniquement géologiques : en plus de l'indication des 
terrains doivent figurer aussi les exploitations en activité ou non dans tous les secteurs économiques. Les informations sont relevées sur deux cartes de Cassini ${ }^{36}$, dont l'une sera conservée par l'ingénieur et l'autre envoyée à l'administration des mines pour y être contrôlée avant de faire graver la carte définitive ${ }^{37}$. La carte d'Henri Résal pour le département du Jura en 1864 (EMP 6275), fournit un très bel exemple de ces minutes manuscrites. Comme pour la carte générale de la France, chaque campagne sur le terrain est accompagnée de la collecte d'échantillons et de fossiles qui sont envoyés au musée de l'École pour enrichir la collection départementale. Parfois des plans précis des lieux de collecte sont alors dressés comme celui des " carrières de pierre à plâtre de Monsieur Leparie sise terroir d'Annet. Lieux-dits les Griffes, Clos Leroy et les Prévalles » (plan EMP, sans cote), où ont été collectés six échantillons d'arbre fossilisé, mentionnés dans le catalogue 1411 de la collection départementale du Musée de minéralogie.

$\mathrm{Si}$ une unification des légendes est tentée entre 1830 et 1865, les cartes départementales restent très disparates dans leur forme, leur échelle et leur degré de précision. À partir de la fin des années 1860, une autre entreprise, celle de la carte géologique détaillée de la France, viendra les remplacer. Un premier ensemble d'une vingtaine de feuilles est proposé lors de l'Exposition universelle de 1855 par A. Dufrénoy et L. Élie de Beaumont, puis un second ensemble d'une soixantaine de feuilles au $1 / 80000^{e}$ sur le fonds topographique de la nouvelle carte d'état-major, à l'Exposition de $1867^{38}$. Ces expériences concluantes conduisent à l'établissement d'un Service de la carte géologique de la France, sous la direction d'Élie de Beaumont, dont le décret de création est signé par Napoléon III en 1868. Ce service sera actif dans les locaux de l'École des mines - jusqu'à sa fusion avec le BRGM en 1968 - dans un nouveau bâtiment dont on conserve les plans parmi les archives $\mathrm{du}$ fonds ancien. L'activité cartographique pour la géologie de la France prend toute son ampleur à cette période pour le Corps des mines. Une nouvelle vision beaucoup plus centralisée et surtout normée de la carte géologique prend le pas sur les monographies locales. Cette volonté de normalisation procède d'un mouvement beaucoup plus général en ce dernier quart du xIx ${ }^{\mathrm{e}}$ siècle : l'unification des codes de représentation cartographique est ainsi discutée lors du premier Congrès géologique international à Paris en $1878^{39}$. La couverture de la carte géologique de la France est complète à plus de $95 \%$ en 1914. Les séries conservées à la bibliothèque, première édition et bien souvent $2^{e}$ et $3^{e}$ éditions, permettent ainsi de percevoir l'évolution des techniques de représentation et des modes de production, comme d'ailleurs l'ensemble des cartes géologiques que nous venons d'évoquer, depuis la représentation par point jusqu'aux aplats représentatifs des différents terrains. Mais le Service n'a pas que des fonctions cartographiques : il se voit bientôt confier aussi d'autres missions comme l'examen des projets d'adduction d'eau potable des communes ou encore dès 1917, une mission de prospection minière : la carte, si elle reflète et accompagne les avancées scientifiques, garde toujours une portée concrète et utilitaire forte, au sein d'une administration destinée au service de l'État. 
La cartothèque de l'École des mines de Paris est remarquable par la régularité et la continuité de sa constitution tout au long de ses deux siècles d'existence. Moins pour le nombre de ses documents ou leur caractère unique, à quelques exceptions près puisque les cartes produites par le Corps des mines étaient destinées le plus souvent à être largement diffusées, c'est pour sa situation au sein d'un ensemble documentaire avec lequel elle est en interaction forte, que la cartothèque mérite toute notre attention. Avec le fonds ancien imprimé et manuscrit, les archives de la bibliothèque et du musée, la collection d'instruments scientifiques, les cartes sont l'un des témoins privilégiés de l'histoire et des fonctions du Corps des mines, un ensemble à haute valeur patrimoniale.

\section{Notes}

1. En 2004, les cartes contemporaines ont été transférées à la bibliothèque de Fontainebleau où l'École a un autre centre sur lequel sont implantés notamment les laboratoires de géosciences.

2. Martine Illaire, Cécile Souchon, « Les cartes et plans au service d'une action : administration, gestion et aménagement du territoire ", in Isabelle LABoulAIs (dir.), Les usages des cartes (XVII ${ }^{e}$ XIX ${ }^{e}$ s.) pour une approche pragmatique des productions cartographiques, Strasbourg, Presses universitaires de Strasbourg, 2008, p. 187.

3. Isabelle LABoulAIs, La Maison des mines. La genèse révolutionnaire d'un corps d'ingénieurs civils (1794-1814), Rennes, Presses universitaires de Rennes, 2012, p. 170-171.

4. Il s'agit cependant ici de documents d'étude : les exemplaires annotés qui ont servi aux ingénieurs des mines sont conservés aux Archives nationales (série F14) ou encore dans les archives départementales.

5. La bibliothèque en possède deux exemplaires dont l'un, de 1780 , est complet, cotes $\mathrm{F}^{\circ}$ Res 71 à 73.

6. Ce dépouillement des cartes n'est disponible pour le moment que dans le fichier papier du catalogue de l'établissement.

7. Ernest Heinrich von DECHEN, Geognostische übersichts-Karte Deutschland, Frankreich, England, Berlin, 1839.

8. Les cartes de ce dernier sont entrées à la bibliothèque par legs en 1892. Elles sont bien identifiées par leur conditionnement dans des boîtes en forme de livre portant la mention « Fondation Rivot ». Certaines sont assez originales par rapport au reste du fonds comme la Carte des Iles britanniques et d'une partie de la France septentrionale dressée pour la lecture des cuvres de Walter Scott (1834).

9. Federico de Botella, Mapa geologico de Espana y Portugal, Madrid, 1879. Carte dédicacée, $\mathrm{n}^{\circ}$ inv. 6784.

10. Jules Marcou, A geological map of the United States and the British provinces, 1853, $\mathrm{n}^{\circ}$ inv. 2058.

11. Michel Armatte, "Théorie et ingénierie économiques : deux siècles de contribution des ingénieurs des mines ", in Anne-Françoise Garçon, Bruno Belhoste (dir.), Les ingénieurs des mines cultures, pouvoirs, pratiques, Paris, Institut de la gestion publique et du développement économique (Histoire économique et financière $\mathrm{XIX}^{\mathrm{e}}$ $x x^{\mathrm{e}}$ siècles), 2012, p. 65. Un cours sur les chemins de fer est aussi institué dès 1848 . 
12. Nicolas VERDIER, «Modeler le territoire : les ingénieurs des Ponts et leurs usages de la carte (fin XVIII ${ }^{\mathrm{e}}$-début XIX ${ }^{\mathrm{e}}$ siècle) ", in Isabelle LABoulaIs (dir.), Les usages des cartes (XVIII'-XIX ${ }^{e}$ siècles)..., op. cit., p. 52.

13. Gilles Thомаs, « L'enseignement de la topographie souterraine à Paris (partie 1) ", Revue XYZ, no 130, 2012, p. 58.

14. Programme des cours de l'École impériale des mines, Paris, 1869. La topographie est bien autonome par rapport aux autres " cours spéciaux ", mais ne prenant pas le rang de cours, elle conserve le titre de «leçons ».

15. Cotes EST n 37 à 62.

16. Voir la collection en ligne dans la bibliothèque numérique patrimoniale : [https://patrimoine.mines-paristech.fr/Instruments], consulté le $30 / 11 / 2017$.

17. Mémoire d'Henri Peslin, p. 59, cote M 1857 (633).

18. Cote J 1877 (597), voir en ligne : [https:// patrimoine.mines-paristech.fr/ark: / 25652 / bLDLiG], consulté le 30/11/2017.

19. Catalogue en ligne des archives et manuscrits de l'enseignement supérieur, [http:/ /www. calames.abes.fr/pub /\#details?id=FileId-2031], consulté le 30/11/2017.

20. Près de 200 Journaux et mémoires de MM. les Elèves sont actuellement disponibles en ligne. Chaque année sont numérisés et mis en ligne environ une trentaine de mémoires, par ensemble géographique. Voir la collection : [https://patrimoine.mines-paristech.fr/Journaux_de_voyage], consulté le 30/11/2017.

21. « Arrêté du même comité, du 18 messidor, concernant les Inspecteurs, Ingénieurs et Elèves des mines ", Journal des mines, $n^{\circ} 1$, Vendémiaire de l'an III (septembre - octobre 1794).

22. L'atlas est consultable en ligne sur la bibliothèque numérique : [https://patrimoine. mines-paristech.fr/document/Atlas_Sarre_1810], consulté le 30/11/2017.

23. Loi du 21 avril 1810 concernant les Mines, les Minières et les Carrières.

24. Lettre de M. Defline, directeur général des Mines domaniales françaises du bassin de la Sarre, au directeur de l'École des mines, 7 mai 1924 (Archives de la bibliothèque).

25. Lettre du 21 août 1925 adressée au Ministre des Travaux publics (Archives de l'École).

26. William Sмітн, A delineation of the strata of England and Wales, with part of Scotland, 1815.

27. Alexandre Brongniart, Georges Cuvier, Description géologique des environs de Paris, Paris, Edmond d'Ocagne, 1835 (EMP, 17664/225).

28. L'ensemble de ces archives est disponible sur le site de la bibliothèque numérique de l'École des mines de Paris : [https://patrimoine.minesparistech.fr/Elie_de_Beaumont_archives_dossier1], consulté le 30/11/2017.

29. Lettre de Becquey de Beaupré, directeur général des Ponts et Chaussées et des mines à Élie de Beaumont, 25 avril 1823, Archives Élie de Beaumont, EMP, dossier I, 10.

30. Lettres de Brochant de Villiers à Élie de Beaumont, 5 juillet 1825, EMP, dossier I, 19, 20 et 21.

31. Jean GAUDANT, « La carte géologique de la France (1841) », Géochroniques, nº 88, 2003, p. 20.

32. Claude Beaumont, "Le Corps des mines et la géologie, deux siècles de relations ", Travaux du Comité français d'Histoire de la Géologie, Comité français d'Histoire de la Géologie, 2010, $3^{\text {e }}$ série (tome 24), p. 153-172.

33. Alexis LegrAND, Circulaire $d u$ directeur général des Ponts et chaussées et des mines aux préfets, 30 août 1835 .

34. Isabelle LABoulAIs, «Aux origines de la carte géologique de France : retour sur les productions cartographiques du Corps des mines au cours du premier XIX ${ }^{e}$ siècle ", in Anne-Françoise Garçon, Bruno Belhoste (dir.), op.cit., p. 28-29.

35. Numérisées dans le cadre du projet Histmap en 2005 en collaboration avec le centre Alexandre Koyré, par Pierre SAvaton et Marie-Noëlle MaIsonneuve [http://histmap. huma-num.fr/cartesgeologiquesfrance/index. php)], ces documents sont aussi accessibles sur le site de la bibliothèque patrimoniale numérique : [https://patrimoine.mines-paristech.fr/ Cartes_g\%C3\%A9ologiques_XIXe], consulté le $30 / 11 / 2017$.

36. Avec les fonds topographiques des cartes de Cassini, l'Atlas national de la France en départements par Chanlaire (1830) servira de base également pour les statistiques départementales. Cf. M. Illaire, C. Souchon, " Les cartes et plans au service d'une action... », art. cité, p. 217 et 220. L'un et l'autre de ces documents se trouvent à la bibliothèque de l'École des mines.

37. Voir entre autres les commentaires d'Élie de Beaumont à propos de la carte de la Seine et Marne par Sénarmont réalisée sur différentes feuilles de Cassini, qui lui ont été adressées pour appréciation (avril 1839). Archives Élie de Beaumont, EMP, dossier II, 36.

38. René MÉdionI, « Les cartes géologiques en France : une aventure de plus de deux siècles », in Jean Gaudant (dir.), L'essor de la géologie française, Paris, Presses des Mines, 2009, p. 205-207.

39. Philippe LagnY, « Paris 1878 : le premier congrès géologique international ", Géochroniques, no 88,2003 , p. 40. 